\title{
Zur Digitalisierung der mittelalterlichen Handschriften des Germanischen Nationalmuseums (GNM)
}

\author{
Daniela Meidlinger
}

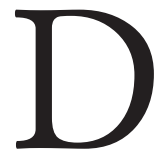
ie Museumsbibliothek zählt formell zum Programmbereich Forschungsservice, sie ist aber zugleich eine der $23 \mathrm{Samm-}$ lungen des Museums. ${ }^{1}$ Unter den rund 150.000 Bänden des Altbestands (bis Erscheinungsjahr I850) ragt die Gruppe der 3380 Handschriften hervor mit Meisterwerken wie dem Codex aureus Epternacensis ([Tresor] Hs ${ }_{156142)^{2}}$, der Melusine-Handschrift (Hs 4028) ${ }^{3}$, dem Trojanerkrieg von Konrad von Würzburg (in: Hs 998) ${ }^{4}$ oder dem Beutelbuch bzw. Stundenbuch des Hieronymus Kress ([Tresor] Hs I723I) ${ }^{5}$. Im Rahmen eines Forschungsprojekts ${ }^{6}$ wurde im Jahr 2012 der finanzielle Grundstein für den Aufbau der Digitalen Bibliothek ${ }^{7}$ des GNM gelegt. Aus der Programmpauschale des Projekts wurde nicht nur ein leistungsfähiger Buchscanner (bookznet Cobra) erworben, es wurde auch ein Mitarbeiter finanziert, der eine eigene Software namens „TOC-Admin“ entwickelte, ein System zur Erfassung, Verwaltung und Erschließung von Buchdigitalisaten für die Digitale Bibliothek des GNM. Nachdem Hardund Software bereitstanden, fuhr man mit der Digitalisierung des Bestandes an mittelalterlichen Handschriften fort. Anhand der gedruckten Bestandskataloge der mittelalterlichen Handschriften von Lotte Kurras ${ }^{8}$ und Hardo Hilg9, die diese in den I970er und i98oer Jahren mit finanzieller Unterstützung der DFG erstellt hatten, wurden die dort verzeichneten 567 Werke der Reihe nach von den studentischen Hilfskräften gescannt. ${ }^{\text {Io }}$ Die so für die Digitalisierung ausgehobenen Bücher wurden direkt im Anschluss für den OnlineKatalog ${ }^{\text {II }}$ katalogisiert, sodass schon Ende 20I3 der
Scan- und erste Erfassungsprozess abgeschlossen war. $\mathrm{Zu}$ dieser Zeit konnten die Benutzer*innen die Digitalisate nur mit Hilfe eines PCs im Lesesaal der Bibliothek einsehen. Das schonte die Originale, da sie nicht mehr zwangsläufig bei jeder Anfrage ausgehoben werden mussten, machte den Bestand allerdings nicht für einen größeren Benutzerkreis sichtbar. Die Veröffentlichung der mittelalterlichen Handschriften in der Digitalen Bibliothek stand noch aus. Im Jahr 2016 wurde daher beschlossen, diesen wertvollen Bestand so schnell wie möglich online verfügbar zu machen. Kalkuliert wurde eine Gesamtlaufzeit des Projekts von circa vier Jahren (es sollte als Teil der offiziellen Digitalisierungsstrategie des Museums vor der kommenden Evaluierung im Frühjahr 202I unbedingt abgeschlossen sein). Zuerst wurden alle Scans in die Digitale Bibliothek gestellt und vollständig mit Metadaten versehen. ${ }^{\mathrm{I} 2}$ Als Vorlage zur Erfassung der Titel- und Strukturdaten wurden auch hier jeweils die gedruckten Kataloge zur Hand genommen. ${ }^{13}$ Das jeweilig zu erfassende Werk wurde dem eigens dafür angelegten Projekt „Handschriften Mittelalter“ zugeordnet. Und erst in einem zweiten Schritt folgte die Katalogisierung der Reproduktionen. Grund dafür war, dass die Katalogisierung in der Regel deutlich mehr Zeit in Anspruch nimmt als die Aufnahme eines Digitalisats in die Digitale Bibliothek allein. Das gilt insbesondere für Handschriftenfragmente, die nur einer kurzen Bearbeitungszeit im hauseigenen Erfassungstool der Digitalen Bibliothek bedürfen, deren Katalogisierung mitunter aber zeitaufwendiger ist. Vor allem auch deshalb, weil 
mit der Anbindung an den Südwestdeutschen Bibliotheksverbund (SWB) sowohl das Exemplar des Originals als auch die elektronische Reproduktion im lokalen sowie im Verbundkatalog eigene Katalogaufnahmen erhalten. Ein Vorteil ist zudem, dass der Vorgang gegen Ende des Projekts auf diese Weise durch die Verteilung auf mehrere Mitarbeiter*innen der Katalogisierung beschleunigt werden kann. Erfahrungen aus bereits abgeschlossenen Projekten ${ }^{14}$ führten $\mathrm{zu}$ dieser Entscheidung. Zusätzlich wurden die Datensätze der Reproduktionen im Lokalkatalog mit eigens dafür angelegten Serien verknüpft. ${ }^{\text {Is }}$ Parallel zur Online-Stellung wurden nach und nach die Uniform Resource Identifier (URI) der jeweiligen Digitalisate auch an den ,Handschriftencensus gemeldet. ${ }^{16}$

Zum Abschluss des Projekts 2019 wurden die mittelalterlichen Handschriften der Paul Wolfgang Merkel'schen Familienstiftung digitalisiert. Diese Sammlung geht auf die Bibliothek der Welser zu Neunhof zurück und wird als Dauerleihgabe in der Bibliothek verwahrt. ${ }^{17}$ Einer Veröffentlichung in der Digitalen Bibliothek wurde dankenswerterweise zugestimmt, sodass auch diese Handschriften als Digitalisat der Wissenschaft zur Verfügung stehen. Damit sind alle mittelalterlichen Handschriften aus dem Bestand der Bibliothek des Germanischen Nationalmuseums online in der Digitalen Bibliothek einzusehen und über die verschiedenen Kataloge und den „Handschriftencensus' recherchierbar. Statt der ursprünglich geplanten vier Jahre waren letztlich nur drei Jahre notwendig, um das Projekt abzuschließen.

Die Digitale Bibliothek der Museumsbibliothek legt Wert auf Funktionalität, und sie wächst kontinuierlich. Die erfreulich hohen Zugriffszahlen, insbesondere aus den Veröffentlichungsjahren der mittelalterlichen Handschriften $2017^{18}$ und $2018^{19}$, spornen an, weitere Bestände online zu stellen. Nach Abschluss der Digitalisierung des Bestandes an mittelalterlichen Handschriften wurde im letzten Jahr auf Anregung des Mediävisten und Archivars Klaus Graf der sogenannte „Handschriften-Kapselkatalog“ gescannt und online in die Digitale Bibliothek gestellt. ${ }^{20}$

Der im I9. Jahrhundert handschriftlich erstellte Katalog erfasst und beschreibt einen Großteil der 3380 Handschriften der Bibliothek, darunter auch die frühneuzeitlichen, für die im Germanischen Nationalmuseum noch keine detaillierten Katalogaufnahmen vorliegen. Freilich kann ein Zettelkatalog die wissenschaftliche Erschließung und Katalogisierung dieses Bestands nicht ersetzen, er ist aber besser als nichts. Derzeit werden auch die nach 1500 entstandenen Handschriften, die im Kapselkatalog beschrieben wurden, der Reihe nach gescannt, katalogisiert und in die $\mathrm{Di}^{-}$ gitale Bibliothek gestellt. Zeitgleich wird auch an der Online-Stellung des Bestandes an circa 1000 Inkunabeln gearbeitet. Eine vollumfängliche Digitalisierung und Katalogisierung anderer bedeutender Sondersammlungen der Bibliothek, wie die der Vorlagenwerke und die der historischen Naturwissenschaften, bleiben einstweilen Desiderate.

Ein erreichbares Ziel dagegen ist die schnellstmögliche Online-Platzierung aller bereits gescannten Werke. Darüber hinaus werden die Werke auch weiterhin an verschiedene Portale gemeldet, um Reichweite und Sichtbarkeit der Rara-Bestände weiter zu erhöhen. Angestrebt wird ferner die ständige Verbesserung des digitalen Bibliotheksauftritts, beispielsweise durch Kommentarfunktionen, die Verknüpfung der Normdaten mit der Gemeinsamen Normdatei (GND) oder die Einführung einer Texterkennungsfunktion (OCR). Damit soll die Zugänglichkeit der Sammlung der Museumsbibliothek weiter verbessert werden. Alle beschriebenen Arbeiten wurden aus Eigenmitteln finanziert. 


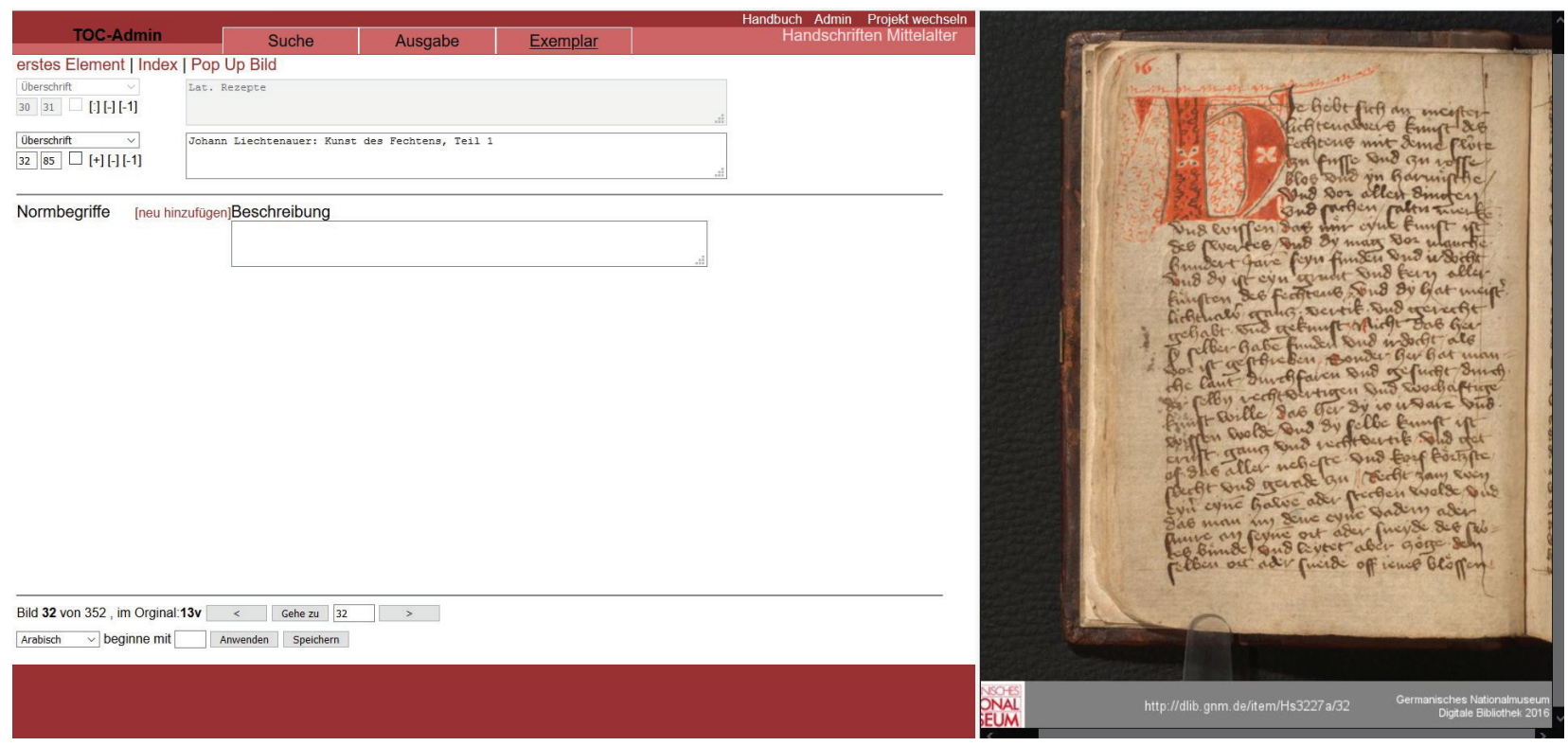

Abb. I: Beispiel für die Strukturdatenerfassung einer Handschrift im Tool „TOC-Admin“

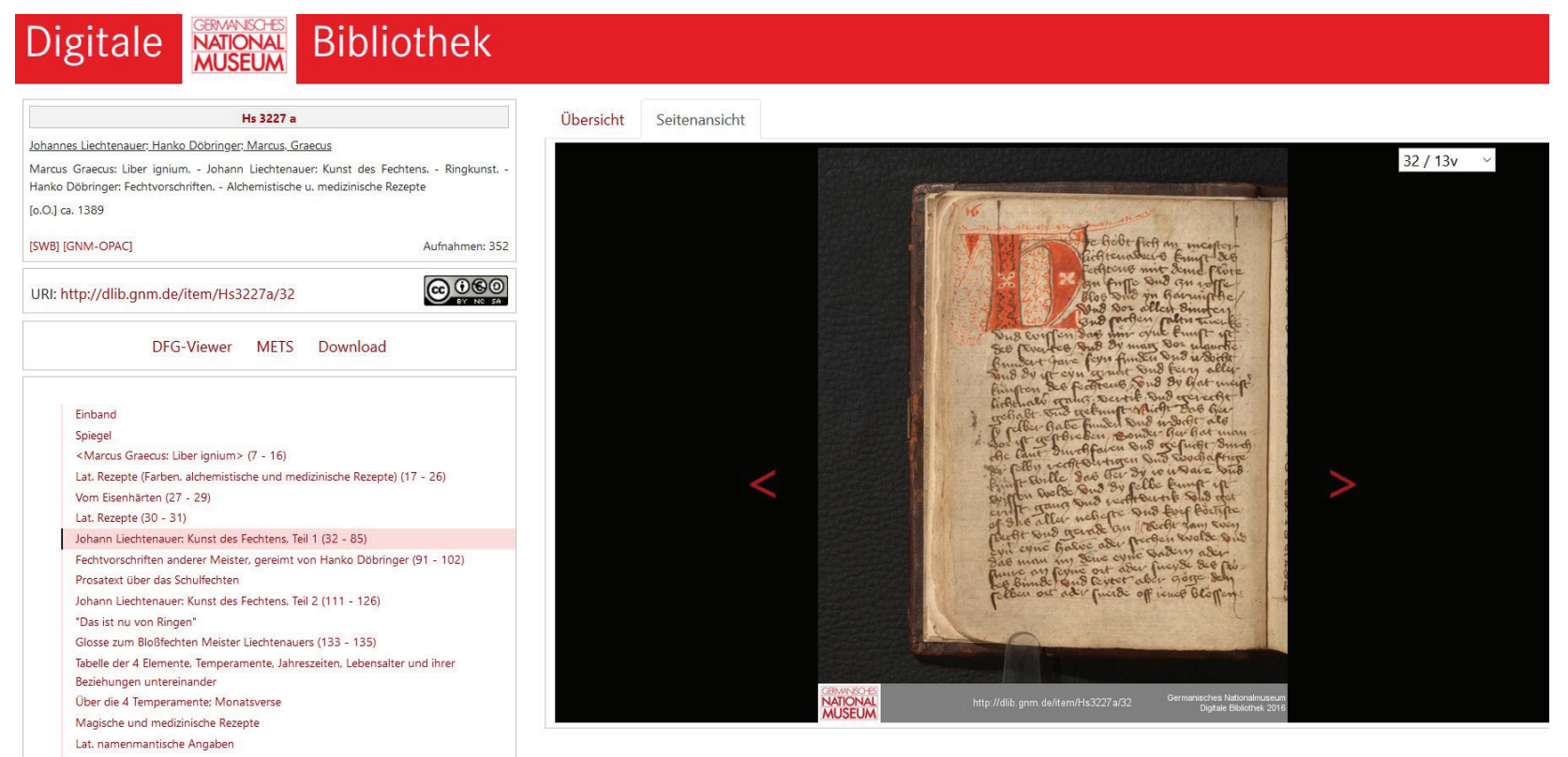

Abb. 2: Darstellung der Bsp.-Handschrift ,Hs 3227 a in der Digitalen Bibliothek <http://dlib.gnm.de/item/Hs3227a/32 (16.04.2020)>

\section{Kontakt}

Daniela Meidlinger

Germanisches Nationalmuseum · Bibliothek | Katalogisierung · Digitale Bibliothek · Kornmarkt I 90402 Nürnberg E-Mail: d.meidlinger@gnm.de

\section{Anmerkungen}

I <https://www.gnm.de/sammlungen/ueberblick-sammlungen/> (I6.04.2020).

$2<$ http://dlib.gnm.de/item/Hsis6I42> (I6.04.2020).

3 <http://dlib.gnm.de/item/Hs4028> (I6.04.2020). 
4 <http://dlib.gnm.de/item/Hs998> (I6.04.2020).

5 <http://dlib.gnm.de/item/Hsi723I> (I6.04.2020).

6 Das Forschungsprojekt „Archäologische Forschungen in Deutschland, Österreich und der Schweiz von der Auflösung des Alten Reichs bis I852“" wurde von der Deutschen Forschungsgemeinschaft (DFG) gefördert und der Bibliothek des Germanischen Nationalmuseums betreut (Projektlaufzeit: 20IO-20I2). Nähere Informationen zu dem Forschungsprojekt finden sich unter: <https://www.gnm.de/forschung/archiv-forschungsprojekte/archaeologische-forschungen/> (I6.04.2020).

7 Startseite der Digitalen Bibliothek: <http://dlib.gnm.de> (I6.04.2020).

8 Lotte Kurras, Die deutschen mittelalterlichen Handschriften: Teil ı. Die literarischen und religiösen Handschriften. Anhang: Die Hardenbergschen Fragmente, Wiesbaden I974.

Lotte Kurras, Die deutschen mittelalterlichen Handschriften: Teil 2. Die naturkundlichen und historischen Handschriften. Rechtshandschriften. Varia, Wiesbaden I980.

9 Hardo Hilg, Die lateinischen mittelalterlichen Handschriften: Teil I. Hs I7a-2292I, Wiesbaden I983. Hardo Hilg, Die lateinischen mittelalterlichen Handschriften: Teil 2. Hs 22922-198390, Anhang, Wiesbaden I986.

Io Wie bei den zuvor erfassten Werken wurde mit einer Farbtiefe von 24 Bit und einer Aufösung von 400 dpi digitalisiert. Die erzeugten Bilddateien wurden als Master in „TIFF uncompressed“ und als Derivate im JPG-Format archiviert.

II Bis zu diesem Zeitpunkt waren die Handschriften nur über die Kartei- und einen sogenannten Kapselkatalog erfasst. Der Online-Katalog, siehe: <https://webopac.gnm.de> (ı6.04.2020).

I2 Zunächst wurden die deutschen mittelalterlichen Handschriften im TOC-Admin erfasst. Nach Ablauf von einem Jahr waren so alle 307 in diesen Katalogen verzeichneten Handschriften in der Digitalen Bibliothek aufgenommen. Daran anschließend folgte die Online-Stellung der 219 lateinischen mittelalterlichen Handschriften.

I3 Vgl. Kurras [Anm. 8] und Hilg [Anm. 9].

I4 Im Vorfeld (ca. zwischen 2012 und 20I6) wurden bereits die Projekte der Nürnberger Handschriften der Frühen Neuzeit (ca. Ioo Werke) und der Inkunabelunikate (28 Stück) abgeschlossen. Die Digitalisate sind in der Digitalen Bibliothek <http://dlib.gnm.de> (I6.04.2020) unter „Handschriften Norica“ und unter „Inkunabeln“ einzusehen.

I5 Vorteile einer solchen Reihe sind die Zitierfähigkeit der digitalisierten Werke eines Projekts, die vereinfachte Statistikabfrage und thematische Recherchemöglichkeit. Ein Beispiel ist die Reihe „Deutsche mittelalterliche Handschriften der Bibliothek des Germanischen Nationalmuseums“, siehe GNM-Onlinekatalog: <http://webopac.gnm. $\mathrm{de} / \mathrm{aDISWeb} / \mathrm{app}$ ?service=direct/o/Home/\$DirectLink\&sp=SOPAC\&sp=SAKoo421687> (ı6.04.2020).

I6 Die Handschriften der Bibliothek des Germanischen Nationalmuseums sind unter folgender Adresse einzusehen: <http://www.handschriftencensus.de/hss/Nuernberg\#bib2> (ı6.04.2020).

17 Einzusehen sind die I2 Digitalisate in der Digitalen Bibliothek unter dem Projekt „Sammlung Merkel“, siehe <http://dlib.gnm.de> (I6.04.2020) oder unter: <http://www.handschriftencensus.de/hss/Nuernberg\#bib2> (I6.04.2020).

I8 Im Jahr 20I7 waren es durchschnittlich 52.750 Zugriffe im Monat auf die Inhalte der Digitalen Bibliothek.

I9 Im Jahr 2018 waren es durchschnittlich 70.453 Zugriffe im Monat auf die Inhalte der Digitalen Bibliothek. $20<$ http://dlib.gnm.de/item/HsKaps> (I6.04.2020). 